\title{
ISOSPECTRAL FLOW IN LOOP ALGEBRAS AND QUASIPERIODIC SOLUTIONS OF THE SINE-GORDON EQUATION ${ }^{\dagger}$
}

\author{
J. HARnAD ${ }^{1}$ AND M.-A. Wisse ${ }^{2}$
}

\begin{abstract}
The sine-Gordon equation is considered in the hamiltonian framework provided by the Adler-Kostant-Symes theorem. The phase space, a finite dimensional coadjoint orbit in the dual space $\mathfrak{g}^{*}$ of a loop algebra $\mathfrak{g}$, is parametrized by a finite dimensional symplectic vector space $W$ embedded into $\mathfrak{g}^{*}$ by a moment map. Real quasiperiodic solutions are computed in terms of theta functions using a Liouville generating function which generates a canonical transformation to linear coordinates on the Jacobi variety of a suitable hyperelliptic curve.
\end{abstract}

\section{Introduction}

Quasiperiodic flows and finite-gap solutions of the sine-Gordon equation

$$
\frac{\partial^{2} u}{\partial x^{2}}-\frac{\partial^{2} u}{\partial t^{2}}=m^{2} \sin (u), m \in \mathbf{R}
$$

have been studied by many authors and derived in a variety of ways $[\mathbf{K K}, \mathbf{C}, \mathbf{M}$, FM, DN, Da, AA, P, FFS, Sm]. Real solutions for two gaps were identified in $[\mathbf{D N}]$ and for any number of gaps in [Da] using Baker-Akhiezer functions. In [FM, AA] the flow was explicitly linearized on the Jacobi variety of a hyperelliptic Riemann surface. A Liouville generating function leads to the linearization in [AA], where

\footnotetext{
${ }^{1}$ Department of Mathematics and Statistics, Concordia University, Montréal, P.Q. and Centre de Recherches Mathématiques, Université de Montréal, C.P. 6128-A, Montréal, P.Q. , Canada H3C 3J7

${ }^{2}$ Département de mathématiques et de statistique, Université de Montréal, C.P. 6128-A, Montréal, P.Q., Canada H3C 3J7

${ }^{\dagger}$ Research supported in part by the Natural Sciences Engineering Research Council of Canada and the Fonds FCAR du Québec
} 
quasiperiodic solutions to equation (1.1) are determined in terms of the integrated flows of a completely integrable finite-dimensional Hamiltonian system. However, the recovery of real solutions remains incomplete in $[\mathbf{F M}]$ and $[\mathbf{A A}]$ and the unifying rôle of the loop algebra $\widetilde{\mathfrak{s u}}(2)$ or the Adler-Kostant-Symes (AKS) theorem [A, K, Sy] does not appear.

In this paper we shall obtain such solutions using a general approach based upon moment maps from finite dimensional symplectic vector spaces into loop algebras as developed in [AHP, AHH1, AHH2]. The AKS theorem and the Liouville-Arnold integration method in the context of loop algebras are central to this approach.

As shown in [AHP], finite dimensional coadjoint orbits $\mathcal{O}$ in loop algebras may be parametrized by symplectic vector spaces via such moment map embeddings. Equations of the type (1.1) are recovered as compatibility conditions for a pair of Hamiltonian Lax equations on $\mathcal{O}$, as given by the AKS theorem. The flow on $\mathcal{O}$ generates, as usual, a flow of line bundles defined on the underlying invariant spectral curve. The divisor coordinates defining these line bundles give a system of Darboux coordinates on $\mathcal{O}$ according to the general scheme developed in [AHH2]. A Liouville generating function yields the canonical transformation to linear coordinates on the Jacobi variety of the spectral curve via the Abel map.

The new element here, relative to the generic case [AHH2], is the passage to a hyperelliptic curve that is not exactly the spectral curve of the flow in $\mathcal{O}$, but rather a quotient by an involutive automorphism, extended by an additional two branch points. The quotienting is necessary because the structure of the Lax pairs implies that the flow is on the twisted loop algebra $\widehat{\mathfrak{s u}}(2)^{*}$, while the additional branch points are necessary to raise the genus of the curve so as to identify the Jacobi variety with the LiouvilleArnold torus. It also becomes clear in this approach that real solutions of (1.1) are equivalent to the choice of a real submanifold in $\mathcal{O}$ corresponding to a coadjoint orbit of the twisted loop algebra $\widehat{\mathfrak{s u}}(2)^{+}$.

\section{Darboux Coordinates for the Sine-Gordon Equation}

First we apply in detail the moment map embedding method developed in [AHP] to the case of the loop algebra $\widetilde{\mathfrak{s u}}(2)$, in order to obtain suitable "Cartesian" coordinates on rational coadjoint orbits. Consider the vector space $\mathbf{C}^{2 \times 2}$ consisting of $2 \times 2$ complex matrices $F$. Let $F_{i}=\left(x_{i}, y_{i}\right), i=1,2$ be the rows of $F$. A real symplectic structure on $\mathrm{C}^{2 \times 2}$ is given by

$$
\widetilde{\Omega}=d F_{2} \wedge d \bar{F}_{1}^{\mathbf{T}}+d \bar{F}_{2} \wedge d F_{1}^{\mathbf{T}}
$$

Now consider the submanifold

$$
\widetilde{\mathcal{M}}=\left\{F \in \mathbf{C}^{2 \times 2}: F_{1} \neq 0, F_{2} \neq 0, F_{2} \bar{F}_{1}^{\mathbf{T}}=0\right\}
$$


This is not a symplectic manifold, but on $\widetilde{\mathcal{M}}$ we have an action of $\mathbf{C}^{*}$ given by

$$
h(F)=\left(\begin{array}{c}
h F_{1} \\
\bar{h}^{-1} F_{2}
\end{array}\right), \quad h \in \mathbf{C}^{*}
$$

which preserves the restriction $\left.\widetilde{\Omega}\right|_{\widetilde{\mathcal{M}}}$ of the symplectic form $(2.1)$ to $\widetilde{\mathcal{M}}$ and whose orbits are the null leaves of $\left.\widetilde{\Omega}\right|_{\widetilde{\mathcal{M}}}$. Thus $\widetilde{\Omega}$ projects to a symplectic form on the quotient manifold $\widetilde{\mathcal{M}} / \mathbf{C}^{*}$. The submanifold $\widetilde{W}$ of $\widetilde{\mathcal{M}}$ given by

$$
\widetilde{W}=\left\{F \in \widetilde{\mathcal{M}} \mid y_{1}=\bar{x}_{2}, y_{2}=-\bar{x}_{1}\right\}
$$

is transversal to the $\mathbf{C}^{*}$-orbits, intersecting each in 2 points, giving a two-sheeted cover of $\widetilde{\mathcal{M}} / \mathbf{C}^{*}$, which is identified with the quotient $\widetilde{W} / \mathbf{Z}_{2}$ of $\widetilde{W}$ by the $\mathbf{Z}_{2}$ action $\pm:\left(x_{1}, x_{2}\right) \mapsto \pm\left(x_{1}, x_{2}\right)$. Thus, restricting $(2.1)$ to $\widetilde{W}$, the manifold $\widetilde{\mathcal{M}} / \mathbf{C}^{*} \equiv \widetilde{W} / \mathbf{Z}_{2}$ inherits the symplectic structure

$$
\left.\widetilde{\omega}\right|_{\widetilde{W}}=2(d \gamma \wedge d \bar{\varphi}+d \bar{\gamma} \wedge d \varphi)
$$

where $(\varphi, \gamma)= \pm\left(x_{1}, x_{2}\right)$, which is well defined on $\widetilde{\mathcal{M}} / \mathbf{C}^{*} \equiv \widetilde{W} / \mathbf{Z}_{2}$. This is just the Marsden-Weinstein reduction at the zero level set of the moment map $F_{2} \bar{F}_{1}^{\mathbf{T}}$ generating the action (2.3).

Now, considering $\mathbf{C}^{(2 N) \times 2}$ as the Cartesian product of $N$ copies of $\mathbf{C}^{2 \times 2}$, with coordinates $\left(\begin{array}{c}F_{2 i-1} \\ F_{2 i}\end{array}\right)=\left(\begin{array}{cc}x_{2 i-1} & y_{2 i-1} \\ x_{2 i} & y_{2 i}\end{array}\right)$ in each $2 \times 2$ block, $\widetilde{\Omega}$ generalizes to the following symplectic form on $\mathbf{C}^{(2 N) \times 2}$ :

$$
\Omega=\sum_{i=1}^{N}\left(d F_{2 i} \wedge d \bar{F}_{2 i-1}^{\mathbf{T}}+d \bar{F}_{2 i} \wedge d F_{2 i-1}^{\mathbf{T}}\right)
$$

On the submanifold

$$
\mathcal{M}=\left\{F \in \mathbf{C}^{(2 N) \times 2} \mid F_{2 i-1} \neq 0, F_{2 i} \neq 0, F_{2 i} \bar{F}_{2 i-1}^{\mathbf{T}}=0, i=1, \ldots, N\right\}
$$

a symplectic action of $\left(\mathbf{C}^{*}\right)^{N}$ is defined by the action $(2.3)$ of $\mathbf{C}^{*}$ on each $2 \times 2$ block $\left(\begin{array}{c}F_{2 i-1} \\ F_{2 i}\end{array}\right)$. The analogue of $\widetilde{W}(2.4)$ is given by

$$
W=\left\{F \in \mathcal{M} \mid y_{2 i-1}=\bar{x}_{2 i}, y_{2 i}=-\bar{x}_{2 i-1}, i=1, \ldots, N\right\} .
$$

Restricting (2.6) to $W$ determines the following symplectic form on the quotient manifold $\mathcal{M} /\left(\mathbf{C}^{*}\right)^{N} \equiv W /\left(\mathbf{Z}_{2}\right)^{N}$ :

$$
\omega=2 \sum_{i=1}^{N}\left(d \gamma_{i} \wedge d \bar{\varphi}_{i}+d \bar{\gamma}_{i} \wedge d \varphi_{i}\right)
$$


where $\left(\varphi_{i}, \gamma_{i}\right)= \pm\left(x_{2 i-1}, x_{2 i}\right), i=1, \ldots, N$.

Let $\widetilde{\mathfrak{s u}}(2)$ be the loop algebra consisting of differentiable maps from a circle $K$ centered at the origin of the complex $\lambda$-plane into $\mathfrak{s u}(2)$ with Lie bracket evaluated, as usual, pointwise. There is a splitting into the direct sum of subalgebras

$$
\widetilde{\mathfrak{s u}}(2)=\widetilde{\mathfrak{s u}}(2)^{+} \oplus \widetilde{\mathfrak{s u}}(2)^{-},
$$

where $\widetilde{\mathfrak{s u}}(2)^{+}$is the subalgebra of loops in $\mathfrak{s u}(2)$ which extend holomorphically inside $K$, while $\widetilde{\mathfrak{s u}}(2)^{-}$is the subalgebra of loops $X(\lambda)$ which extend holomorphically outside $K$ and normalized by the condition $X(\infty)=0$. The Lie algebra $\widetilde{\mathfrak{s u}}(2)\left(\right.$ resp., $\widetilde{\mathfrak{s u}}(2)_{0}^{-}=$ $\{X \in \mathfrak{s u}(2) \mid X$ extends holomorphically outside $K$, with finite limit $X(\infty)\})$ is densely embedded into $\widetilde{\mathfrak{s u}}(2)^{*}$ (resp., $\widetilde{\mathfrak{s u}}(2)^{+*}$ ) via the nondegenerate, ad-invariant bilinear form on $\widetilde{\mathfrak{s u}}(2)$ given by

$$
<X, Y>:=\oint_{K} \frac{\operatorname{tr}(X(\lambda) Y(\lambda))}{\lambda} d \lambda, \quad X, Y \in \widetilde{\mathfrak{s u}}(2)
$$

Henceforth no notational distinction will be made between elements of $\widetilde{\mathfrak{s u}}(2)^{*}$ (resp., $\widetilde{\mathfrak{s u}}(2)^{+*}$ ) and elements of $\widetilde{\mathfrak{s u}}(2)$ (resp., $\left.\widetilde{\mathfrak{s u}}(2)_{0}^{-}\right)$.

Fix $N$ complex numbers $\alpha_{i}, i=1, \ldots, N$ in the interior of $K$. We define an injective moment map $\widetilde{J}$ on $\mathcal{M} /\left(\mathbf{C}^{*}\right)^{N}$, which is locally identified with $W$, by

$$
\widetilde{J}\left(\varphi_{1}, \gamma_{1}, \ldots, \varphi_{N}, \gamma_{N}\right)=\lambda \sum_{i=1}^{N} \frac{\left(\begin{array}{cc}
-\bar{\gamma}_{i} \varphi_{i} & \bar{\gamma}_{i}^{2} \\
-\varphi_{i}^{2} & \bar{\gamma}_{i} \varphi_{i}
\end{array}\right)}{\alpha_{i}-\lambda}+\frac{\left(\begin{array}{cc}
\gamma_{i} \bar{\varphi}_{i} & \bar{\varphi}_{i}^{2} \\
-\gamma_{i}^{2} & -\gamma_{i} \bar{\varphi}_{i}
\end{array}\right)}{\bar{\alpha}_{i}-\lambda} \in \widetilde{\mathfrak{s u}}(2)^{+*}
$$

The fact that this is really a Poisson map with respect to the Lie-Poisson structure on $\widetilde{\mathfrak{s u}}(2)^{*}$ and that, viewed as a moment map, it generates a Hamiltonian action of the corresponding loop group $\widetilde{S U}(2)^{+}$on $W$, follows from the general results developed in [AHP]. It is also easily verified that the image of this map coincides with a coadjoint orbit in $\widetilde{\mathfrak{s u}}(2)^{+*}$. To obtain real solutions of the sine-Gordon equation, however, we must restrict to a submanifold of $W$ whose image lies in the dual of the "twisted" subalgebra $\widehat{\mathfrak{s u}}(2)$ of $\widetilde{\mathfrak{s u}}(2)$, consisting of fixed points of the involutive automorphism

$$
\sigma(X)(\lambda)=\left(\begin{array}{cc}
1 & 0 \\
0 & -1
\end{array}\right) X(-\lambda)\left(\begin{array}{cc}
1 & 0 \\
0 & -1
\end{array}\right), \quad X \in \widetilde{\mathfrak{s u}}(2)
$$

Analogously to (2.10) we have a splitting of $\widehat{\mathfrak{s u}}(2)=\widehat{\mathfrak{s u}}(2)^{+} \oplus \widehat{\mathfrak{s u}}(2)^{-}$, as well as embeddings $\widehat{\mathfrak{s u}}(2) \hookrightarrow \widehat{\mathfrak{s u}}(2)^{*}$ (resp., $\left.\widehat{\mathfrak{s u}}(2)_{0}^{-} \hookrightarrow \widehat{\mathfrak{s u}}(2)^{+*}\right)$ via $(2.11)$, where $\widehat{\mathfrak{s u}}(2)_{0}^{-}$is defined analogously to $\widetilde{\mathfrak{s u}}(2)_{0}^{-}$.

In order that condition (2.13) be satisfied by the image of $\widetilde{J}$, the $\alpha_{i}$ 's have to come in pairs of opposite sign. There are two possibilities: either $\alpha_{i}$ has a nonzero 
real part, in which case we need both $\alpha_{i}$ and $-\alpha_{i}$, or $\alpha_{i}$ is purely imaginary. We may reorder these constants so that $\alpha_{i+p}=-\alpha_{i}, i=1, \ldots, p$ for the $\alpha_{i}$ 's with a real part, and $\alpha_{j}=\sqrt{-1} \beta_{j}, j=2 p+1, \ldots, N$ for the purely imaginary constants $\alpha_{j}$. On $W$ we must then impose the further constraints (cf. [AHP, sec. 5])

$$
\begin{gathered}
\varphi_{i+p} \bar{\gamma}_{i+p}=\varphi_{i} \bar{\gamma}_{i}, \quad \bar{\gamma}_{i+p}^{2}=-\bar{\gamma}_{i}^{2}, \quad \bar{\varphi}_{i+p}^{2}=-\bar{\varphi}_{i}^{2}, \quad i=1, \ldots, p \\
\varphi_{j} \bar{\gamma}_{j}=-\bar{\varphi}_{j} \gamma_{j}, \quad \varphi_{j}^{2}=-\gamma_{j}^{2}, \quad j=2 p+1, \ldots, N .
\end{gathered}
$$

Solving these constraints we obtain

$$
\begin{gathered}
\varphi_{i+p}=\sqrt{-1} \varphi_{i}, \quad \gamma_{i+p}=\sqrt{-1} \gamma_{i}, \quad i=1, \ldots, p \\
\varphi_{j}=\sqrt{-1} \gamma_{j}, \quad j=2 p+1, \ldots, N .
\end{gathered}
$$

Let $\widehat{W}$ be the submanifold of $W$ given by constraints (2.14a.b) and (2.15a,b). The restriction $\hat{\omega}$ of $\omega$ to $\widehat{W}$ is

$$
\hat{\omega}=4 \sum_{i=1}^{p}\left(d \gamma_{i} \wedge d \bar{\varphi}_{i}+d \bar{\gamma}_{i} \wedge d \varphi_{i}\right)+4 \sqrt{-1} \sum_{j=2 p+1}^{N} d \bar{\gamma}_{j} \wedge d \gamma_{j},
$$

showing that $\hat{\omega}$ is of maximal rank, and $\widehat{W}$ a symplectic subspace.

In terms of the reduced coordinates, the restriction $\widehat{J}$ of $\widetilde{J}$ to $\widehat{W}$ is

$$
\widehat{J}=2 \lambda\left(\begin{array}{cc}
b(\lambda) & c(\lambda) \\
-\bar{c}(\bar{\lambda}) & -b(\lambda)
\end{array}\right)
$$

with $b(\lambda), c(\lambda)$ given by

$$
\begin{aligned}
& b(\lambda)=\lambda \sum_{i=1}^{p}\left(\frac{-\varphi_{i} \bar{\gamma}_{i}}{\alpha_{i}^{2}-\lambda^{2}}+\frac{\bar{\varphi}_{i} \gamma_{i}}{\bar{\alpha}_{i}^{2}-\lambda^{2}}\right)+\sqrt{-1} \lambda \sum_{j=2 p+1}^{N} \frac{\left|\gamma_{j}\right|^{2}}{\beta_{j}^{2}+\lambda^{2}} \\
& c(\lambda)=\sum_{i=1}^{p}\left(\frac{\alpha_{i} \bar{\gamma}_{i}^{2}}{\alpha_{i}^{2}-\lambda^{2}}+\frac{\bar{\alpha}_{i} \bar{\varphi}_{i}^{2}}{\bar{\alpha}_{i}^{2}-\lambda^{2}}\right)-\sqrt{-1} \sum_{j=2 p+1}^{N} \frac{\beta_{j} \bar{\gamma}_{j}^{2}}{\beta_{j}^{2}+\lambda^{2}} .
\end{aligned}
$$

Thus, $\widehat{J}$ takes values in the dual of the twisted loop algebra $\widehat{\mathfrak{s u}}(2)^{+}$. On $\widehat{\mathfrak{s u}}(2)^{+*}$, define the ring of functions

$$
\begin{aligned}
& \mathcal{F}_{+}=\left\{\Phi \in C^{\infty}\left(\widehat{\mathfrak{s u}}(2)^{+*}\right)|\Phi=\widehat{\Phi}|_{\widehat{\mathfrak{s u}}(2)^{+*}}, \widehat{\Phi} \in C^{\infty}\left(\widehat{\mathfrak{s u}}(2)^{*}\right)\right. \\
& \left.\mu([d \widehat{\Phi}(\mu), X])=0, \forall \mu \in \widehat{\mathfrak{s u}}(2)^{*}, X \in \widehat{\mathfrak{s u}}(2)\right\} .
\end{aligned}
$$


That is, $\mathcal{F}_{+}$consists of the restriction to $\widehat{\mathfrak{s u}}(2)^{+*}$ of the infinitesimally $A d^{*}$-invariant functions on $\widehat{\mathfrak{s u}}(2)$. It follows from the AKS theorem that the Hamiltonians in $\mathcal{F}_{+}$ commute in the Lie-Poisson structure of $\widehat{\mathfrak{s u}}(2)^{+*}$. Choose Hamiltonians $H_{\xi}, H_{\eta} \in \mathcal{F}_{+}$ defined by

$$
\begin{aligned}
& H_{\xi}(X)=\frac{1}{2} \operatorname{tr}\left(\frac{a(\lambda)}{\lambda^{2}}(X(\lambda)+\lambda Y)^{2}\right)_{0} \\
& H_{\eta}(X)=-\frac{1}{2} \operatorname{tr}\left(\frac{a(\lambda)}{\lambda^{2 N}}(X(\lambda)+\lambda Y)^{2}\right)_{0}
\end{aligned}
$$

where $a(\lambda)=\prod_{i=1}^{p}\left[\left(\lambda^{2}-\alpha_{i}^{2}\right)\left(\lambda^{2}-\bar{\alpha}_{i}^{2}\right)\right] \prod_{j=2 p+1}^{N}\left(\lambda^{2}+\beta_{j}^{2}\right)$ and $Y=\left(\begin{array}{cc}0 & -1 \\ 1 & 0\end{array}\right)$. According to the AKS theorem, Hamilton's equations for

$$
\mathcal{N}(\lambda)=\widehat{J}+\lambda Y
$$

are given by

$$
\begin{aligned}
\frac{d}{d \xi} \mathcal{N} & =-\left[d H_{\xi}(\mathcal{N})_{-}, \mathcal{N}\right] \\
\frac{d}{d \eta} \mathcal{N} & =\left[d H_{\eta}(\mathcal{N})_{+}, \mathcal{N}\right]
\end{aligned}
$$

where the "+" (resp., "-") subscript denotes projection to $\widehat{\mathfrak{s u}}(2)^{+}$(resp., $\left.\widehat{\mathfrak{s u}}(2)^{-}\right)$. Setting

$$
\mathcal{L}(\lambda)=\frac{a(\lambda)}{\lambda} \mathcal{N}(\lambda)=\lambda^{2 N-1} \mathcal{L}_{0}+\lambda^{2 N-2} \mathcal{L}_{1}+\cdots+\lambda^{0} \mathcal{L}_{2 N-1}+a(\lambda) Y,
$$

we have

$$
\begin{aligned}
& d H_{\xi}(\mathcal{N})_{-}=\frac{1}{\lambda}\left(\mathcal{L}_{2 N-1}+a(0) Y\right) \\
& d H_{\eta}(\mathcal{N})_{+}=\mathcal{L}_{0}+\lambda Y
\end{aligned}
$$

and the Lax equation for $\mathcal{L}(\lambda)$ is obtained by multiplication of $(2.22)$ by $\frac{a(\lambda)}{\lambda}$.

The flow leaves invariant the spectral curve with affine part given by

$$
\mathcal{P}(\lambda, z)=\operatorname{det}(\mathcal{L}(\lambda)-z I)=0 \text {. }
$$

Expanding (2.25) and using the fact that the rank of the residues of $\widehat{J}$ is equal to 1 , we may write

$$
\begin{gathered}
\mathcal{P}(\lambda, z)=z^{2}+a(\lambda) P(\lambda)=0 \\
P(\lambda)=P_{0}+\lambda^{2} P_{1}+\cdots+\lambda^{2 N-2} P_{N-1}+\lambda^{2 N} .
\end{gathered}
$$


The coefficients $P_{i}$ are all in $\mathcal{F}_{+}$; in particular,

$$
H_{\xi}=-P_{0}, \quad H_{\eta}=P_{N-1}
$$

The $P_{i}$ are thus constant along the flows of all Hamiltonians in $\mathcal{F}_{+}$. We may choose a level set

$$
P_{0}=\operatorname{det}\left(\mathcal{L}_{2 N-1}+a(0) Y\right) \equiv \frac{m^{2}}{16} \in \mathbf{R},
$$

which implies that we may write

$$
\mathcal{L}_{2 N-1}+a(0) Y=\frac{m}{4}\left(\begin{array}{cc}
0 & e^{\sqrt{-1} u} \\
-e^{-\sqrt{-1} u} & 0
\end{array}\right),
$$

where $u$ is real. From (2.29) we see that $u$ may be written in terms of the coordinates $\left(\varphi_{i}, \gamma_{i}, i=1, \ldots, p ; \gamma_{j}, j=2 p+1, \ldots, N\right)$ as follows:

$$
e^{\sqrt{-1} u}=a(0)(c(0)-1) .
$$

From the compatibility conditions for equations (2.22) it now follows that $u$ satisfies equation (1.1), where $\xi=x+t$ and $\eta=x-t$.

[Remark. Equation (1.1) may equivalently be viewed as the compatibility conditions for the $x$ and $t$ flows determined by the two Hamiltonians $H_{x}:=-H_{\xi}-H_{\eta}, H_{t}:=H_{\xi}-H_{\eta}$.]

\section{Quasiperiodic Solutions for the Sine-Gordon equation}

The hyperelliptic spectral curve (2.25) has genus $g=2 N-1$. However, it is invariant under the involution $(z, \lambda) \mapsto(z,-\lambda)$, and hence is a two-sheeted covering of the hyperelliptic curve $C^{\prime}$, with genus $N-1$, whose affine part given is by

$$
z^{2}+\tilde{a}(E) \widetilde{P}(E)=0
$$

where $\widetilde{P}\left(\lambda^{2}\right)=P(\lambda)$ and $\tilde{a}\left(\lambda^{2}\right)=a(\lambda), \lambda^{2}=E$. We also define functions $\tilde{b}, \tilde{c}$ by $\tilde{b}(E)=b(\sqrt{E}), \tilde{c}(E)=c(\sqrt{E})$. In order to apply the Jacobi inversion method we shall need a hyperelliptic curve $\widetilde{C}$ of genus $N$ with affine part given by

$$
\tilde{z}^{2}+E \tilde{a}(E) \widetilde{P}(E)=0 .
$$

This is obtained from $C^{\prime}$ by setting $\tilde{z}=z \lambda$, which adds branch points at $E=0$ and $E=\infty$. Following the general method for the introduction of "spectral Darboux coordinates" developed in [AHH2], we define on $\widetilde{C}$ a divisor of degree $N$ with coordinates $\left(E_{\mu}, \zeta_{\mu}\right)_{\mu=1, \ldots, N}$. The $E_{\mu}$ are given by the equation

$$
\tilde{c}\left(E_{\mu}\right)-1=0
$$


or, equivalently

$$
\sum_{i=1}^{p}\left(\frac{\alpha_{i} \bar{\gamma}_{i}^{2}}{\alpha_{i}^{2}-E}+\frac{\bar{\alpha}_{i} \bar{\varphi}_{i}^{2}}{\bar{\alpha}_{i}^{2}-E}\right)-\sqrt{-1} \sum_{j=2 p+1}^{N} \frac{\beta_{j} \bar{\gamma}_{j}^{2}}{\beta_{j}^{2}+E}-1=-\frac{\prod_{\mu=1}^{N}\left(E-E_{\mu}\right)}{\tilde{a}(E)} .
$$

These may be viewed as complex hyperelliptic coordinates if the $\left(\varphi_{i}, \gamma_{i}, i=1, \ldots, p\right.$; $\left.\gamma_{j}, j=2 p+1, \ldots, N\right)$ are interpreted as Cartesian coordinates on the submanifold of $\mathcal{M} /\left(\mathbf{C}^{*}\right)^{N}$ determined by the constraints $(2.14 \mathrm{a}, \mathrm{b})$. The canonically conjugate coordinates $\zeta_{\mu}$ are defined by

$$
\zeta_{\mu}=\sqrt{-\frac{\widetilde{P}\left(E_{\mu}\right)}{E_{\mu} \tilde{a}\left(E_{\mu}\right)}}=-\frac{2 \tilde{b}\left(E_{\mu}\right)}{\sqrt{E_{\mu}}}
$$

i.e. by the eigenvalues of the matrix $\frac{\mathcal{N}(\lambda)}{\lambda^{2}}$ at $\lambda^{2}=E_{\mu}$. Comparing with (2.30) we see that

$$
\begin{gathered}
e^{\sqrt{-1} u}=-\prod_{\mu=1}^{N}\left(-E_{\mu}\right) \\
u=-\sqrt{-1}\left(\sum_{\mu=1}^{N} \ln \left(-E_{\mu}\right)-\pi\right) .
\end{gathered}
$$

Proposition 3.1. The coordinates $\left(E_{\mu}, \zeta_{\mu}\right)_{\mu=1, \ldots, N}$ form a Darboux coordinate system on the coadjoint orbit passing through $\mathcal{N}(\lambda)$. The corresponding symplectic form is

$$
\omega_{\mathcal{N}}=\sum_{\mu=1}^{N} d E_{\mu} \wedge d \zeta_{\mu}=-d \theta
$$

Proof. Computing the differentials of the residues of (3.4) and summing up we find, using (3.5),

$$
4 \sum_{i=1}^{p}\left(\varphi_{i} d \bar{\gamma}_{i}-\gamma_{i} d \bar{\varphi}_{i}\right)+4 \sum_{j=2 p+1}^{N} \gamma_{j} d \bar{\gamma}_{j}=\sum_{\mu=1}^{N} \zeta_{\mu} d E_{\mu}=\theta
$$

[Remark: This result could also have been obtained as in [AHH2], by computing implicitly the Lie-Poisson brackets of $\left(E_{\mu}, \zeta_{\mu}\right)$ on the coadjoint orbit passing through $\mathcal{N}(\lambda) \cdot]$ 
The restriction of (3.8) to the invariant level sets $P_{i}=c_{i}=$ const., $i=0, \ldots, N-1$ is identically zero; i.e. this defines a Lagrangian submanifold. Hence in a neighborhood of $P_{i}=c_{i}$, the one-form $\theta=d S$ may be integrated on the leaves of the Lagrangian foliation as usual to yield the Liouville generating function

$$
S\left(P_{i}, E_{\mu}\right)=\sum_{\mu=1}^{N} \int_{E_{0}}^{E_{\mu}} \sqrt{-\frac{\widetilde{P}(E)}{E \tilde{a}(E)}} d E,
$$

where $E_{0} \in \widetilde{C}$ is a suitably chosen base point. Derivation of $S$ with respect to the $P_{i}$ 's gives the conjugate coordinates $Q_{i}$, in terms of which the flows of the Hamiltonians in the ring generated by the $P_{i}$ 's are linear.

$$
Q_{i}=\frac{\partial S}{\partial P_{i}}=-\frac{1}{2} \sum_{\mu=1}^{N} \int_{E_{0}}^{E_{\mu}} \frac{E^{i}}{\sqrt{-E \tilde{a}(E) \widetilde{P}(E)}} d E .
$$

Hamilton's equations for $H_{\xi}, H_{\eta}$ are thus integrated to give

$$
\sum_{\mu=1}^{N} \int_{E_{0}}^{E_{\mu}} \frac{E^{i}}{\sqrt{-E \tilde{a}(E) \widetilde{P}(E)}} d E=C_{i}+2 \delta_{i, 0} \xi-2 \delta_{i, N-1} \eta
$$

on the Jacobi variety of $\widetilde{C}$.

If the holomorphic differentials

$$
\omega_{i}=\frac{E^{i}}{\sqrt{-E \tilde{a}(E) \widetilde{P}(E)}} d E
$$

were normalized, the left hand side of (3.12) would just be the Abel map. Since the $\omega_{i}$ form a basis of holomorphic differentials for $\widetilde{C}$, choosing a basis $\left(a_{i}, b_{i}\right)_{i=1, \ldots, N}$ of $H_{1}(\widetilde{C}, \mathbf{Z})$ such that $a_{i} \cdot a_{i}=b_{i} \cdot b_{i}=0, a_{i} \cdot b_{j}=\delta_{i j}$, the matrix $M$ of integrals over the $a$-cycles of the differentals $\omega_{i}$,

$$
M_{i j}=\oint_{a_{i}} \omega_{j}
$$

is invertible. Multiplying equation (3.12) by $M^{-1}$, the flow for equation (1.1) is linearized on the Jacobi-variety of the curve $\widetilde{C}$ via the Abel map

$$
A\left(p_{1}+\cdots+p_{N}\right)=U \eta+V \xi+B,
$$

where $p_{\mu} \in \widetilde{C}$ are the points with coordinates $\left(\zeta_{\mu}, E_{\mu}\right)$, and $U, V, B$ are constant vectors in $\mathbf{C}^{N}$ obtained by applying $M^{-1}$ to the vectors with components $-2 \delta_{i, N-1}, 2 \delta_{i, 0}$ and $C_{i}$, respectively, appearing in equation (3.12). It remains to explicitly compute $u(\xi, \eta)$ as given by equation (3.7) from this linear flow. Let $\Theta$ be the theta function corresponding to the hyperelliptic curve $\widetilde{C}$ and $\kappa$, the Riemann constant. From (3.7) follows, in a standard way (cf. [GH, Du, AHH2 (Cor. 1.7)]): 


\section{Proposition 3.2.}

$$
u=-2 \sqrt{-1} \ln \frac{\Theta(A(0)-U \eta-V \xi-B-\kappa)}{\Theta(A(\infty)-U \eta-V \xi-B-\kappa)}+C
$$

where $C$ is an integration constant independant of $\eta$ and $\xi$.

Proof. As usual we consider $\widetilde{C}$ as a two sheeted branched cover of $\mathbf{P}^{1}(\mathbf{C})$ with projection mapping $\pi: \widetilde{C} \rightarrow \mathbf{P}^{1}(\mathbf{C})$ and branch locus $\mathcal{B}$. Let $\left\{a_{i}, b_{i}\right\}, i=1, \ldots, N$ be a basis of $H_{1}(\widetilde{C}, \mathbf{Z})$ with common base point $p_{0}$. Let $\pi\left(a_{i}\right)$ and $\pi\left(b_{i}\right)$ be the projections to $\mathbf{P}^{1}(\mathbf{C})$. We suppose that the $a_{i}$ 's are chosen such that the winding numbers $n\left(\pi\left(a_{i}\right), 0\right)$ and $n\left(\pi\left(a_{i}\right), \infty\right)$ are zero and such that their intersection indices are given by $a_{i} \cdot a_{j}=b_{i} \cdot b_{j}=$ $0, a_{i} \cdot b_{j}=\delta_{i j}$. Consider a polygonization $\Delta$ of $\widetilde{C}$ with respect to $\left(a_{i}, b_{i}\right)$. Assume that $F(E)=\Theta(A(E)-U \eta-V \xi-B-\kappa)$ is not identically zero on $\widetilde{C}$ (which holds for generically chosen $B$ ) and that $E_{\mu} \neq 0, \infty, \mu=1, \ldots, N$. On $\Delta$ choose integration paths $c_{0}$ (resp., $c_{\infty}$ ) from one of the representatives of $p_{0}$ on $\Delta$ (i.e. one of the vertices of $\Delta$ ) to 0 (resp., $\infty$ ). Cut along these paths to obtain a polygonization $\widetilde{\Delta}$. On $\widetilde{\Delta}$ the differential

$$
\varphi=\ln (-E) d \ln F(E)
$$

is well defined and meromorphic. It is easily computed that

$$
\sum_{\mu=1}^{N} \ln \left(-E_{\mu}\right)=\oint_{\partial \widetilde{\Delta}} \varphi
$$

whereas the right hand side of (3.17) yields (see e.g. [GH])

$$
\begin{aligned}
\int_{a_{i}+a_{i}^{-1}} \varphi & =\text { constant in } \eta \text { and } \xi \\
\int_{b_{i}+b_{i}^{-1}} \varphi & =0 \\
\int_{c_{0}+c_{0}^{-1}} \varphi+\int_{c_{\infty}+c_{\infty}^{-1}} \varphi & =2 \pi \sqrt{-1} \ln \frac{\Theta(A(0)-U \eta-V \xi-B-\kappa)}{\Theta(A(\infty)-U \eta-V \xi-B-\kappa)} .
\end{aligned}
$$

Summing up these terms and substituting in (3.7) yields the desired result.

\section{Conclusions:}

The form of the quasiperiodic solutions (3.15) agrees with that obtained by other authors (e.g., [FM, DN]) who have studied the sine-Gordon equation by a variety of methods. The new element presented here is the placing of these solutions entirely 
within the framework of isospectral flows in loop algebras and the AKS theorem. The reality conditions and reductions related to the invariance of the spectral curve under involutions follow naturally in this approach from the use of the twisted loop algebra $\widehat{\mathfrak{s u}}(2)$. The Darboux coordinates leading to a completely separated Liouville generating function $S$ given by (3.10) and the linearization of the flow via the Abel map are seen as illustrative cases of the "spectral Darboux coordinate" method developed in [AHH2].

Acknowledgements: The authors are pleased to acknowledge helpful discussions with J. Tafel relating to the contents of this paper.

\section{REFERENCES}

[A] Adler, M., On a Trace Functional for Formal Pseudo-Differential Operators and the Symplectic Structure of the Korteweg- de Vries Equation, Invent. Math. 50 (1979), 219-248.

[AA] Al'ber, S.J., Al'ber, M.S., Hamiltonian Formalism for Nonlinear Schrödinger Equations and Sine-Gordon Equations, J. London Math. Soc. (2) 36 (1987), 176-192.

[AHH1] Adams, M.R., Harnad, J. and Hurtubise, J., Isospectral Hamiltonian Flows in Finite and Infinite Dimensions II. Integration of Flows, Commun. Math. Phys. 134 (1990), 555-585.

[AHH2] Adams, M.R., Harnad, J. and Hurtubise, J., Darboux Coordinates and LiouvilleArnold Integration in Loop Algebras, CRM-preprint (1991); Coadjoint Orbits, Spectral Curves and Darboux Coordinates, in: "The Geometry of Hamiltonian Systems", ed. T. Ratiu, Publ. MSRI Springer-Verlag, New York (1991); Liouville Generating Function for Isospectral Hamiltonian Flow in Loop Algebras, in: "Integrable and Superintergrable Systems", ed. B. Kuperschmidt, World Scientific, Singapore (1990)

[AHP] Adams, M.R., Harnad, J. and Previato, E., Isospectral Hamiltonian Flows in Finite and Infinite Dimensions I. Generalized Moser Systems and Moment Maps into Loop Algebras, Commun. Math. Phys. 117 (1988), 451-500.

[C] Cherednik, I.V., Reality Conditions in Finite Zone Integration, Dokl. Akad. Nauk SSR 25, no. 5 (1980), 1104-1108.

[Da] Date, E., Multi-Soliton Solutions and Quasi-Periodic Solutions of Nonlinear Equations of Sine-Gordon Type, Osaka J. Math. 19 (1982), 125-158.

[Du] Dubrovin, B.A., Theta Functions and Nonlinear Equations, Russ. Math. Surv. 36 (1981), 11-92.

[DN] Dubrovin, B.A., Natanzon, S.M., Real Two Zone Solutions of the Sine-Gordon Equation, Funkt. Anal. Appl. 16 (1982), 21-33. 
[FFS] Flesh, R., Forest, M.G., Sinha, A., Numerical inverse spectral transform for the periodic sine-Gordon equation: Theta-functions solution and their linearized stability. Physica D 48 (1991), 169-231. D.W., Spectral Theory for the periodic Sine-Gordon Equation: A Concrete Viewpoint, J. Math. Phys. 23 (1982), no. 7, $1248-1277$.

[GH] Griffith, P. and Harris, J., "Principles of Algebraic Geometry", John Wiley, New York, 1978.

[K] Kostant, B., The Solution to a Generalized Toda Lattice and Representation Theory, Adv. Math. 34 (1979), 195-338.

[KK] Kozel, V.A., Kotlyarov, V.P., Almost Periodical Solutions of the Equation $u_{t t}-$ $u_{x x}+\sin (u)=0$, Dokl. Akad. Nauk UkSSR, Ser. A 10 (1978), 878-881 (in Ukrainian).

[M] McKean, H.P., The Sine-Gordon and Sinh-Gordon Equations on the circle, Comm. Pure Appl. Math. 34 (1981), 197-257.

[P] Previato, E. A particle-system model of the sine-Gordon hierarchy, Solitons and coherent structures, Physica D 18, no. 1-3 (1986), 312-314.

[Sm] Smirnov, A.O., Real elliptic solutions of the sine-Gordon equation. Math of the USSR Sbornik 70 (1991), 231-240.

[Sy] Symes, W., Systems of Toda Type, Inverse Spectral Problems and Representation Theory, Invent. Math. 59 (1980), 13-51. 